\title{
Hospitalización por influenza en un Servicio de Pediatría de Santiago de Chile, 2001-2005
}

\author{
Luis E. Vega-Briceño, Lisbeth Platzer M., M. Angéliza Oyarzún A., Katia Abarca V., \\ Dahiana Pulgar B. e lgnacio Sánchez D.
}

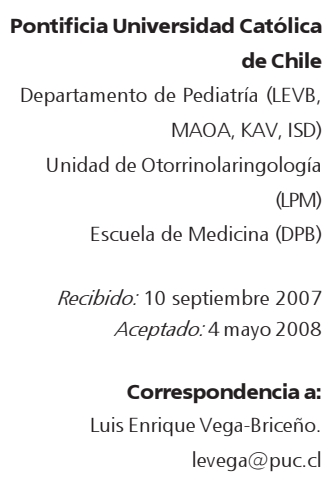

\section{Hospitalization due to influenza in children in Santiago, Chile, 2001-2005}

Background: Influenza cause high hospitalization rates and complications in children. Objective: To describe clinical and epidemiological characteristics of influenza infection in hospitalized children. Patients and methods: In Universidad Católica Hospital, all hospitalizations due to influenza in children aged 15 days to 14 years, ocurring between January 2001 and December 2005 were reviewed. Results: Of a total of 3570 admissions associated with a respiratory illness, $124(3.5 \%)$ were due to influenza, of which $75 \%$ presented between the months of April and June. Median age was 20.5 months (60\% younger than 2 years) and 24\% had an underlying risk factor. Most common symptoms were fever (94\%) and dry cough (61\%) and $75 \%$ of the children required oxygen. The most frequent complication was pneumonia (53\%). The mean duration of hospitalization and oxygen use were 4.4 and 2.5 days respectively. Fifty two children (49.1\%) received an antibiotic and nine children were admitted to intensive care unit. No deaths were recorded. Conclusion: Influenza virus cause serious complications and affects mostly healthy children younger than 2 years.

Key words: influenza, complications, children, hospitalization

Palabras clave: influenza, complicaciones, niños, hospitalización.

\section{Introducción}

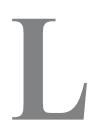
a infección por influenza es considerada una enfermedad emergente que produce, anualmente, un elevado impacto en morbi-mortalidad y costos a nivel mundial ${ }^{1-2}$. Cerca de $30 \%$ de los niños y $5 \%$ de los adultos desarrollan síntomas gripales anualmente; siendo los niños bajo 2 años de edad quienes exhiben las tasas más alta de hospitalización y complicaciones $^{3,4}$. En nuestro país, la influenza tiene una marcada presentación estacional durante la temporada otoño-invierno ${ }^{5,6}$ y desde el año 1983 , está sujeta a notificación mediante un programa de vigilancia en centros centinelas ${ }^{7}$.

Luego de la primera descripción clínica hecha por Hipócrates, el año 412 a.C, se reportaron las primeras pandemias, a partir del siglo XVI de nuestra era ${ }^{1}$. Hoy en día se reconoce que las manifestaciones clínicas dependen de diversos factores como la edad, el estado inmune, las condiciones crónicas de base y la virulencia de la cepa circulante ${ }^{4,8,9}$. Es difícil estimar la verdadera incidencia de la infección por este virus en niños, ya que la mayoría presenta síntomas leves e inespecíficos y no consultan a un servicio de salud; mas aún, se reconoce la dificultad -en ausencia de estudios de laboratorio- de distinguirla de otras infecciones virales o bacterianas, por lo que ha menudo se subestima su presencia o se confunde con infecciones bacterianas lo que conlleva un uso irracional de antibacterianos ${ }^{10,11}$.

Si bien hasta $60 \%$ de estas infecciones pueden ser subclínicas, existen complicaciones graves y eventualmente mortales en niños ${ }^{12,13}$. Así por ejemplo, durante la temporada influenza 2003-2004, en los Estados Unidos de Norteamérica, murieron 143 niños a causa de este virus, $45 \%$ de ellos no tenían condiciones de riesgo asociadas ${ }^{14}$. La elevada tasa de infección y complicaciones en este grupo etario sustentó en el año 2003 la recomendación de la Academia Americana de Pediatría de vacunar rutinariamente contra influenza a los lactantes entre 6 y 24 meses de edad. En nuestro país, esta política fue adoptada a partir del año 2006; con anterioridad, la vacunación en los niños estaba restringida a los grupos de alto riesgo ${ }^{15}$. Nuestro objetivo fue describir el perfil clínico-epidemiológico de los niños hospitalizados por virus influenza durante un período de 5 años en el Servicio de Pediatría del Hospital Clínico de la Pontificia Universidad Católica de Chile.

\section{Pacientes y Métodos}

De manera retrospectiva se identificaron todos los pacientes pediátricos hospitalizados en nuestra insti- 
tución por alguna condición respiratoria durante los años 200 a 2003 y de forma prospectiva, durante los años 2004 y 2005. Para efectos de este reporte se incluyeron sólo aquellos pacientes con confirmación virológica de la infección mediante técnica de inmunofluorescencia directa (IFD) y/o inmunocromatografía (Test pack ${ }^{\circledR}$ ) previamente descritas ${ }^{5}$. Se revisó la ficha médica de cada niño, registrando condiciones crónicas de base, factores de riesgo, síntomas y signos previos, motivo y evolución de la hospitalización, exámenes de laboratorio, complicaciones, tratamientos indicados y condición final al alta.

Definiciones. Neumonía bacteriana: presencia de fiebre $\left(\geq 37,5^{\circ} \mathrm{C}\right.$ axilar o $38^{\circ} \mathrm{C}$ rectal) y crepitaciones pulmonares asociadas a leucocitosis. Hipoxemia: saturación arterial de oxígeno transcutánea $\leq 90 \%$. Alguna condición de base: antecedentes de prematurez, alergia, sibilancias recurrentes, genopatía. Factor de riesgo: presencia de alguna condición que incremente el riesgo de complicaciones derivadas de la infección por influenza (patología cardíaca o respiratoria crónica, enfermedad neurológica, inmunodeficiencias o malignidades hematológicas) ${ }^{13}$. Sibilancias recurrentes: presencia de $\geq 3$ episodios de sibilancias, confirmadas por médico. Sibilancias asociadas a virus: conjunto bronquiolitis (en los menores de 1 año), bronquitis obstructiva o crisis asmáticas. Niño sano: ausencia de las condiciones antes mencionadas.

Para el análisis estadístico, se empleó el paquete computacional SPSS para Windows; considerando todas la variables numéricas o categóricas, siendo las variables numéricas expresadas como medias \pm desviación estándar o medianas y rangos. Para establecer comparaciones se empleó la prueba de $\mathrm{t}$ de Student $\mathrm{y}$ $\chi^{2}$, considerando significativas aquellas con $\mathrm{p}<0,05$.

\section{Resultados}

Durante el período 2001-2005 se hospitalizaron 124 niños por influenza confirmada, siendo el virus influenza A, el más frecuente (90\%). En 12 niños (10\%) se encontró co-infección con otros agentes respiratorios: VRS (5), virus parainfluenza (3), Bordetella pertussis (2) y Mycoplasma pneumoniae (2). Se solicitó Test pack® en 51 niños, resultando negativo en 16; sin embargo, la IFD posterior fue positiva en todos ellos. Un paciente presentó IFD negativa aunque la inmunocromatografía fue posteriormente positiva. El inicio de la temporada influenza fue variable y osciló entre la semana 12 y 25 . El $75 \%$ de todas las hospitalizaciones ocurrieron durante abril y junio, especialmente durante la temporada $2004(95 \%)^{5}$. El virus influenza A circuló preferentemente en otoño (79\%). De los 5 años estudiados, destaca el mayor número de casos de influenza durante los años 2001 y 2004 (Tabla 1)

Se revisó la ficha médica de $109(88 \%)$ pacientes. La edad promedio fue 20,5 meses (rango: 15 días-14,5 años), $55 \%$ de género masculino. Distribución por edad: $14 \%$ bajo 6 meses, $46 \%$ entre 6 y 24 meses; $30 \% 2$ a 5 años y $10 \%$ sobre 5 años de edad. Quince por ciento asistía a sala cuna. Previo a la hospitalización, 30\% de los niños habían recibido antibacterianos. Veintiséis (24\%) niños tenían al menos un factor de riesgo identificado: bronquiolitis obliterante o daño pulmonar postviral (22), enfermedad neurológica (7), cardiopatía (5), enfermedad muscular en ventilación mecánica domiciliaria (3) y alguna malignidad hematológica (2); 34/109 niños $(31 \%)$ tenían al menos dos condiciones crónicas de base simultáneas. Por el carácter retrospectivo de este estudio se desconoce el número verdadero de pacientes vacunados en cada temporada.

Tabla 1. Distribución de hospitalizaciones respiratorias y detección del virus influenza durante el período 2001-2005

\begin{tabular}{|c|c|c|c|c|c|}
\hline & 2001 & 2002 & 2003 & 2004 & 2005 \\
\hline Número total de hospitalizaciones de causa respiratoria & 950 & 856 & 701 & 558 & 505 \\
\hline IFD ○ IC realizados & $436(46 \%)$ & $467(55 \%)$ & $361(52 \%)$ & $315(56 \%)$ & $361(71 \%)$ \\
\hline IFD ○ IC positivos a algún agente (\%) & $s / d$ & $s / d$ & $s / d$ & $200(64 \%)$ & $271(75 \%)$ \\
\hline Casos de influenza & 45 & 6 & 18 & 40 & 15 \\
\hline Influenza $A / B$ & $42 / 3$ & $6 / 0$ & $17 / 1$ & $35 / 5$ & $13 / 2$ \\
\hline Cepa influenza A aislada (ISP) & $\mathrm{H} 3 \mathrm{~N} 2$ & H1N 1 & $\mathrm{H} 3 \mathrm{~N} 2$ & $\mathrm{H} 3 \mathrm{~N} 2$ & $\mathrm{H} 3 \mathrm{~N} 2$ \\
\hline Tasa de vacunación (\%) & 0 & 0 & 0 & 12,5 & 20 \\
\hline Semana epidemiológica de inicio de temporada & 25 & 14 & 18 & 12 & 21 \\
\hline Circulación influenza en otoño (\%) & 58 & 83 & 22 & 93 & 86 \\
\hline Circulación de influenza en invierno (\%) & 40 & 17 & 72 & 7 & 14 \\
\hline
\end{tabular}




\begin{tabular}{|lcc|}
\hline \multicolumn{3}{|c|}{ Tabla 2. Diagnóstico de egreso de 124 nin̄os } \\
hospitalizados por influenza
\end{tabular}

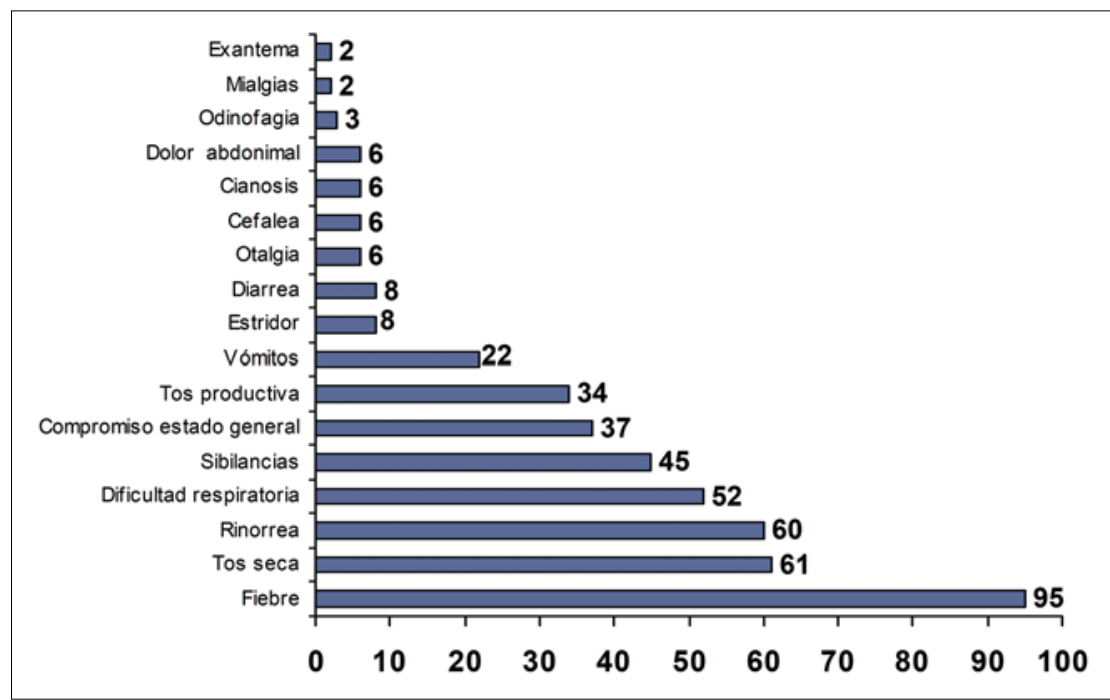

Figura 1. Manifestaciones clínicas de los pacientes pediátricos hospitalizados por influenza.
El promedio (días) de síntomas previo a la hospitalización fue 5,3 3,8. La Figura 1 muestra las manifestaciones clínicas más frecuentes reportadas. La gran mayoría de los niños (84\%) estaba febril al momento del ingreso y $75 \%$ requirió oxígeno (6 niños con cianosis), siendo el principal motivo de hospitalización la hipoxemia. Otras causas incluyeron: síndrome febril, convulsión febril (4 casos asociados a influenza A) y miositis. El promedio (días) de fiebre fue 3,3 $\pm 2,5$. La Tabla 2 muestra los diagnósticos clínicos más frecuentes de egreso. La neumonía bacteriana fue la complicación más frecuente $(53 \%)$ siendo el virus influenza A responsable de $92 \%$ de ellas. Sesenta y cinco por ciento de las hospitalizaciones por influenza A ocurrió en lactantes, no se encontró asociación entre la edad del paciente y el tipo de virus influenza identificado $(\mathrm{p}=0,86)$. La influenza $\mathrm{B}$ se asoció a neumonías intersticiales (5), croup (2), cuadros de sibilancias (2) y síndrome febril (1).

Durante la hospitalización, el requerimiento de oxígeno fue: 61 con $\mathrm{FiO}_{2}$ menor a 40\%, $10 \mathrm{FiO}_{2} 40-80 \%$ y 3 $\mathrm{FiO}_{2}$ mayor a $80 \%$ con un promedio (días) de aporte de oxígeno de 2,5 (rango: 0-12,5). Sesenta y cinco por ciento de los pacientes con factores de riesgo requirió al menos cuatro días oxígeno. Se administró salbutamol y corticoesteroides a 80 y 35 niños, respectivamente. En 88 casos se solicitó radiografía de tórax, siendo informada como normal en 13 de ellos. Los hallazgos radiográficos más frecuentes fueron la presencia de infiltrado intersticial e hiperinsuflación (63\%).

La Tabla 3 muestra las principales características clínicas según edad. Se encontró algunas diferencias estadísticamente significativas en lactantes bajo 6 meses en relación al grupo total: menor duración de la hospitalización $(2,8 \pm 1,2$ días y 4,4 $\pm 3,1$ días $\mathrm{p}=0,045)$ menos días de fiebre y de uso de oxígeno $(\mathrm{p}=0,028)$.

\begin{tabular}{|c|c|c|c|c|}
\hline & Total (n: 109) & $<6$ meses $(n: 15)$ & 6-24 meses ( $n: 50)$ & $>24$ meses $(n: 44)$ \\
\hline Fiebre (días)* & $3,3(0-15)$ & $2,7 \quad(0-8)$ & $5,1 \quad(1-12)$ & $4,9(1-15)$ \\
\hline Hospitalización (días) & $4,4(1-16)$ & $2,8(1-5)$ & $4,2(1-16)$ & $4,9(1-14)$ \\
\hline Uso de oxígeno (\%) & 75 & 54 & 83 & 65 \\
\hline Tiempo de oxígenoterapia (días) & $2,5(0-12,5)$ & $1,8(0-4)$ & $3,0(0-6)$ & $4,1 \quad(0-12,5)$ \\
\hline Uso de antibacterianos (\%) & 53 & 54 & 55 & 50 \\
\hline Consulta previa (\%) & 61 & 38 & 75 & 55 \\
\hline Niño previamente sano (\%) & 43 & 100 & 40 & 37 \\
\hline $\begin{array}{l}\text { * Expresado como media (rango) } \\
\text { * Expresado según el número tot }\end{array}$ & & & & \\
\hline
\end{tabular}


En 92/98 casos se solicitó hemograma y/o PCR al momento de la hospitalización, encontrándose 19 casos con leucocitosis (mayor a $15.000 / \mathrm{mm}^{3}$ ) y 7 con leucopenia (menor a $4.500 / \mathrm{mm}^{3}$ ). La PCR estuvo alterada en $43 / 78$ pacientes $(55 \%)$.

Durante la hospitalización, 52 niños recibieron antibacterianos: 10 tenían factores de riesgo, 18 condiciones crónicas de base y 24 eran sanos. Las indicaciones más frecuentes de su empleo fueron: neumonía sobreinfectada (31), otitis media aguda (8), sospecha de sobre-infección bacteriana por aparición de leucocitosis asociada a fiebre (3), aumento en requerimientos de oxígeno y deterioro clínico (2), otras (4). Catorce niños recibieron amantadina.

Finalmente, 9 niños (4 durante la epidemia 2004) ingresaron a la Unidad de Cuidados Intensivos (UCI) por aumento de los requerimientos de oxígeno y deterioro del estado general (edad promedio 12 meses) con una estancia promedio en UCI de 8 días; en $8 / 9$ se identificó influenza A. Todos presentaron como complicación una neumonía probablemente bacteriana; se identificó co-infección con Bordetella pertussis en 2 casos. No se registraron muertes ni requerimientos de ventilación mecánica en esta serie.

\section{Discusión}

Las infecciones respiratorias preferentemente virales, representan la principal causa de morbilidad en la edad pediátrica en países desarrollados ${ }^{16,17}$. Nuestro estudio mostró que la infección por influenza es una causa importante de hospitalización pediátrica en nuestro medio, siendo responsable de $3,5 \%$ del total de hospitalizaciones respiratorias durante el período de estudio. Si bien la influenza sigue un patrón estacional, cuyo inicio es difícil de predecir ${ }^{16}$, la principal actividad epidemiológica en nuestra serie se registró al inicio de los meses fríos del año (preferentemente junio), aunque este comportamiento fue variable en cada año. En general, nuestros hallazgos son similares a la realidad epidemiológica nacional publicada previamente $\mathrm{e}^{5,6,11}$ y es concordante con lo observado en los países de clima templado ${ }^{17}$.

La mayoría de los niños hospitalizados por influenza tenían bajo dos años de edad; principalmente eran niños previamente sanos sin condiciones de riesgo conocidas. Al igual que en otros estudios, la condición crónica pre-existente más frecuente fue el asma o la presencia de sibilancias recurrentes ${ }^{8,16-18}$. Nuestros hallazgos confirman lo reportado por Neuzil ${ }^{7,18}$ e Izurieta $^{16}$, en relación a que la influenza causa un alto número de hospitalizaciones y complicaciones en lactantes antes sanos, presentando tasas de hospitali- zación tan altas como los adultos sobre 65 años de edad. Nuestro estudio fue realizado en el período previo a la vacunación rutinaria de lactantes sanos en Chile y sus resultados apoyan fuertemente esta recomendación.

Las manifestaciones clínicas más frecuentes de la influenza en niños fueron fiebre, rinorrea, tos seca y dificultad respiratoria, similar a lo reportado por otros autores ${ }^{2,4,17}$. La evolución clásica del adulto con calofríos, odinofagia, cefalea y mialgias no son usuales de reconocer en niños ${ }^{6,12,17}$. Por otra parte, los síntomas gastrointestinales y el compromiso de sistema nervioso central fueron infrecuentes en nuestra serie. La complicación más frecuente fue la neumonía bacteriana; se ha reconocido que este virus predispone a la sobreinfección bacteriana por Streptococcus pneumoniae en niños ${ }^{2,19,20}$. La menor gravedad observada en los niños bajo 6 meses de edad podría explicarse por ausencia de condiciones crónicas de base o de factores de riesgo asociados, inmunización pasiva materna, protección por lactancia o mayor laxitud en la decisión de hospitalización de niños muy pequeños.

Nuestros datos confirman la importancia y necesidad del diagnóstico etiológico de las infecciones virales en niños de manera precoz, implementando métodos rápidos y confiables. Si bien el cultivo viral de secreción nasofaríngea es considerado el estándar de oro, su costo, tiempo e infraestructura necesaria limitan su aplicación ${ }^{6}$. Las técnicas rápidas de diagnóstico (ensayo del tipo inmunocromatografía) tienen menor sensibilidad; sin embargo, fueron propuestas para favorecer medidas de prevención inmediatas, disminuir el uso de antibacterianos innecesarios y la tasa de transmisión nosocomial ${ }^{21-23}$. Tal como lo apoyan nuestros resultado, un resultado negativo no descarta el diagnóstico de influenza, por lo que se recomienda confirmarlo con IFD o cultivo viral ${ }^{1}$. Si bien el diseño de nuestro estudio no permite establecer un análisis detallado del comportamiento de las pruebas diagnósticas (sensibilidad, especificidad, falsos positivos y negativos), nuestros hallazgos sugieren un adecuado rendimiento de la IFD. La RPC ha surgido como un método rápido, sensible y específico de gran utilidad, que promete la pronta tipificación de las cepas circulantes ${ }^{10,24}$. El mejoramiento de las técnicas diagnósticas puede hacer más factible el uso de medicamentos antivirales en las primeras $48 \mathrm{~h}$ del inicio de los sínto$\operatorname{mas}^{11,17}$.

La influenza en niños produce elevadas tasas de hospitalización, complicaciones y uso de antibacterianos $^{2,16,25}$, siendo muchas de ellas prevenibles. La vacunación anual ha demostrado ser segura, bien tolerada y efectiva en niños, protegiendo también a otros segmentos de la población (efecto rebaño), ya que se 
reconoce que los niños tienen un rol importante en la propagación de este virus en la comunidad ${ }^{1,24}$. Muchos padres y algunos médicos, aún creen (erróneamente) que la vacuna está recomendada exclusivamente para los sujetos sobre 65 años de edad o población de riesgo, ya que la influenza es considerada una enfermedad benigna y autolimitada en niños ${ }^{22}$. Probablemente, éste sea el principal obstáculo para la inmunización pediátrica. Reichert y colaboradores, demostraron que la vacunación masiva de escolares, evitó una muerte por cada 420 escolares vacunados ${ }^{26}$, lo que sugiere que la vacunación es una medida costo-efecti$\mathrm{va}^{13,18}$. El desarrollo de nuevas vacunas intranasale ${ }^{27-30}$, puede hacer que la inmunización sea más aceptada por la comunidad, lo que ayudaría a incrementar su cobertura. Considerando que la mayoría de los niños hospitalizados en esta serie tenían menos de dos años, con la recomendación actual de protegerlos con una vacuna eficaz y segura, muchas de estas hospitalizaciones podrían ser evitadas en el futuro ${ }^{28-31}$. Sin embargo, en lactantes bajo 6 meses de edad, la vacunación no está recomendada, por lo que las medidas preventivas deben apuntar a sus contactos o, eventualmente, hacia una recomendación más global en la comunidad ${ }^{17}$.

En conclusión, nuestros datos muestran que la infección por influenza no es una condición benigna, sino que produce un importante impacto en términos de morbilidad, días de hospitalización, uso de oxígeno y complicaciones que requieren ser tratadas con antibacterianos. Mas aún, muchos de estos episodios pudieron haber sido potencialmente evitados con una adecuada estrategia de inmunización ${ }^{31}$. Estos resulta- dos deben ser considerados para brotes epidémicos futuros en nuestro medio.

Agradecimientos: A la Sra. Teresa Azócar por su cuidadoso trabajo en el diagnóstico de infección por virus influenza mediante IFD en el Laboratorio de Virología e Infectología Molecular de la Pontificia Universidad Católica de Chile.

\section{Resumen}

Introducción: El virus influenza produce elevadas tasas de hospitalización y complicaciones en niños. Objetivo: Describir el perfil clínico-epidemiológico de los niños hospitalizados por influenza en un hospital universitario. Pacientes y Métodos: Estudio descriptivo de las hospitalizaciones respiratorias y por influenza entre enero 2001 y diciembre 2005 en el Hospital Clínico de la Pontificia Universidad Católica. Resultados: Se hospitalizaron 3.570 niños por alguna causa respiratoria, $124(3,5 \%)$ correspondieron a influenza confirmada, $75 \%$ ocurrieron entre abril y junio. La edad promedio fue 20,5 meses $(60 \%<2$ años), $24 \%$ tenían algún factor de riesgo identificado. Los síntomas más frecuentes fueron: fiebre $(94 \%)$ y tos seca (61\%). El $75 \%$ presentó hipoxemia, siendo la complicación más frecuente la neumonía (53\%). El promedio (días) de hospitalización y oxígeno fue 4,4 y 2,5 ; respectivamente. En 52/109 se indicaron antibacterianos, 9 niños ingresaron a cuidados intensivos. No hubo fallecidos. Conclusión: La hospitalización por influenza se asoció a serias complicaciones y afecta especialmente a niños sanos menores de 2 años.

\section{Referencias}

1.- Nicholson K, Wood J, Zambon M. Influenza. Lancet 2003; 362: 1733-45.

2.- Neuzil K M, Mellen B G, Wright P F, Mitchel E F, Griffin M R. The effect of influenza on hospitalizations, outpatient visits, and courses of antibiotics in children. N Engl J Med 2000; 342: 225-31.

3.- Kempe A, Daley M, Barrow J, Allred N, Hester N, Beaty BL, et al. Implementation of universal influenza immunization recommendations for healthy young children: Results of a randomized, controlled trial with registry-based recall. Pediatrics 2005; 115: 146-54.

4.- Arostegui K N, Montes M, Pérez-Yarza E G, Sardón O, Vicente D, Cilla G. Características clínicas de los niños hospitalizados por infección por virus influenza. An Pediatr (Barc) 2005; 62: 5-12.

5.- Vega-Briceño L E, Potin M, Bertrand P,
Sánchez I. Infección respiratoria por virus influenza en niños: ¿Que aprendimos durante el 2004? Rev Méd Chile 2005; 133 : 911-8.

6.- Delpiano L, Guillen B, Casado M C. Comportamiento clínico-epidemiológico de la influenza en niños hospitalizados. Rev Chil Infect 2003; 20: 159-65.

7.- Sotomayor V. Boletín de vigilancia en salud pública de Chile. El Vigía 2004; 19: 25-8

8.- Glezen W P, Greenberg S B, Atmar R L, Piedra P A, Couch R B. Impact of respiratory virus infections on persons with chronic underlying conditions. JAMA 2000; 283: 499-505.

9.- Neuzil K M, Wright P F, Mitchel E F, Griffin M R. The burden of influenza illness in children with asthma and other chronic medical conditions. J Pediatr 2000; 137 : 856-64.

10.- Aymard M, Valette M, Luciani J. Burden of influenza in children: preliminary data from a pilot survey network on community diseases. Pediatr Infect Dis J 2003; 22 (Suppl 10): S211-4.

11.- Proyecto Vigilancia de virus respiratorios. http://www.virus.med.puc.cl/ virus_respiratorios/ (accedido el 19 diciembre 2006).

12.- Peltola V, Ziegler T, Ruuskanen O Influenza $\mathrm{A}$ and $\mathrm{B}$ infections in children. Clin Infect Dis 2003; 36: 299-305.

13.- Committee on Infectious Diseases. American Academy of Pediatrics. Reduction of the influenza burden in children. Pediatrics 2002; 110 : 1246-52.

14.- Centers for Disease Control and Prevention (CDC) Advisory Committee on Immunization Practices (ACIP). Recommendations of the ACIP. MMWR Morb Mortal Wkly Rep RR 2004; 53: $1-40$. 
15. - http://www.minsal.cl/ici/pandemiainfluenza/ anexo\%205.pdf (accedido el 04 de mayo del 2007).

16. - Izurieta H S, Thompson W, Kramarz P, Shay D K, Davies R L, DeStefano F, et al. Influenza and the rates of hospitalization for respiratory disease among infants and young children. N Engl J Med 2000; 342; 232-9.

17. - Muñoz F M. Influenza virus infection in infancy and early childhood. Paediatr Respir Rev 2003; 4: 99-104.

18. - Neuzil K M, Zhu Y, Griffin M R, Edwards K M, Thompson J M, Tollefson S J, et al. Burden of interpandemic influenza in children younger than 5 years: a 25 -year prospective study. J Infect Dis 2002; 185: 147-52.

19.- Heikkinen T, Thint M, Chonmaitree $\mathrm{T}$ Prevalence of various respiratory viruses in the middle ear during acute otitis media. N Engl J Med 1999; 340: 260-4.

20. - O'Brien K L, Walters M I, Sellman J, Quinlisk P, Regnery H, Schwartz B, et al. Severe pneumococcal pneumonia in previously healthy children: the role of preceding influenza infection. Clin Infect
Dis 2000; 30: 784-9.

21. - Laundy M, Ajayi-Obe J, Hawrami K, Aitken C, Breuer J, Booy R. Influenza A community acquired pneumonia in east London infants and young children. Pediatr Infect Dis J 2003; 22: 223-7.

22.- Poehling K A, Speroff T, Dittus R S, Griffin M R, Hickson G B, Edwards K M. Predictors of influenza virus vaccination status in hospitalized children. Pediatrics 2001; 108: E99.

23. - Noyola D E, Demmler G J. Effect of rapid diagnosis on management of influenza A infections. Pediatr Infect Dis J 2000; 19: 303-7.

24.- Heininger H. An update on the prevention of influenza in children and adolescents. Eur J Pediatr 2003; 162: 828-36.

25.- Cox N J, Subbarao K. Influenza. Lancet 1999; 354: 1277-82.

26. - Reichert T A, Sugaya N, Glezen W P, Simonsen L, Tashiro M. The Japanese experience with vaccinating schoolchildren against influenza. N Engl J Med 2001; 344 $889-96$
27. - Luce B R, Zangwill K M, Palmer C S, Mendelman P M, Yan L, Wolff M C, et al. Cost-effectiveness analysis of an intranasal influenza vaccine for the prevention of influenza in healthy children. Pediatrics 2001; 108: E24.

28. - O'Brien M A, Uyeki T M, Shay D K, Thompson W W, Kleinmann K, McAdam K, et al. Incidence of outpatient visits and hospitalizations related to influenza in infants and young children Pediatrics 2004; 113: 585-93

29. - Zangwill K M, Belshe R. Safety and efficacy of trivalent inactivated influenza vaccine in young children: a summary for the new era of routine vaccination. Pediatr Infect Dis J 2004; 23: 189-200

30.- Jefferson T, Smith S, Demicheli V, Harnden A, Rivetti A, Di Pietrantonj C. Assessment of the efficacy and effectiveness of influenza vaccines in healthy children: Systematic review. Lancet 2005; 365: 773-80.

31. - Vega-Briceño L E, Abarca K, Sánchez I. Vacuna influenza en niños: estado de arte. Rev Chil Infectol 2006; 23: 164-9. 